\title{
Efeito do manejo da cobertura do solo na maturação da videira Merlot cultivada em região de elevada altitude
}

\begin{tabular}{|c|c|}
\hline Douglas André Wurz & $\begin{array}{l}\text { Universidade do Estado de Santa Catarina, Centro de Ciências Agrárias - } \\
\text { UDESC/CAV, Avenida Luiz de Camões 2090, Bairro Conta Dinheiro, Lages-SC, } \\
\text { 88520-000. } \\
\text { E-mail: douglaswurz@hotmail.com }\end{array}$ \\
\hline José Luiz Marcon Filho & $\begin{array}{l}\text { Vinícola Legado, Rod. Raul Azevedo de Macedo, } 5800 \text { - CEP 83606-482, Campo } \\
\text { Largo-PR. } \\
\text { E-mail: marconfilho_jl@yahoo.com.br }\end{array}$ \\
\hline $\begin{array}{l}\text { Betina Pereira de Bem } \\
\text { Leo Rufato } \\
\text { Aike Anneliese Kretzschmar }\end{array}$ & $\begin{array}{l}\text { Universidade do Estado de Santa Catarina, Centro de Ciências Agrárias - } \\
\text { UDESC/CAV, Avenida Luiz de Camões 2090, Bairro Conta Dinheiro, Lages-SC, } \\
\begin{array}{l}\text { 88520-000. } \\
\text { E-mails: riardoufsc@gmail.com } \\
\text { betadebem@yahoo.com.br } \\
\text { leoruffato@yahoo.com.br } \\
\text { aikeanneliese@yahoo.com.br }\end{array}\end{array}$ \\
\hline
\end{tabular}

Recebido: 10 jul. 2017. Aceito: 22 dez. 2017.

DOI: http://dx.doi.org/10.21674/2448-0479.41.152-158

\section{Resumo}

Tem-se como objetivo deste trabalho comparar a maturação tecnológica e fenólica da videira Merlot em região de elevada altitude de Santa Catarina cultivada em solo coberto por vegetação e solo sem cobertura vegetal. O experimento foi realizado na safra 2014/2015 em um vinhedo comercial situado no munícipio de São Joaquim - Santa Catarina. O delineamento experimental utilizado foi o de blocos ao acaso, com quatro blocos e dez plantas por bloco. Foi utilizado um vinhedo da variedade Merlot, enxertada sobre 'Paulsen 1103'. Os tratamentos consistiram em manter a linha de cultivo com cobertura vegetal natural e manter a linha de cultivo sem cobertura vegetal. Para manter a linha de cultivo sem cobertura vegetal foi realizado capina manual a partir do estágio fenológico virada de cor, sendo esta operação repetida a cada 15 dias até o momento da colheita. Avaliou-se a maturação tecnológica e fenólica das bagas, além da massa de baga e relação casca/baga. Observou-se efeito da cobertura do solo na maturação da videira Merlot, em solo sem cobertura vegetal na linha de cultivo, houve incremento da relação casca/baga, $\mathrm{pH}$ e antocianinas. Portanto o solo sem cobertura vegetal resultou em uma melhor maturação da videira Merlot cultivada em região de elevada altitude.

Palavras-chave: Vitis vinifera L. Antocianinas. Qualidade enológica. Vinhos de altitude.

\section{Abstract \\ Effect of soil cover management on maturation of Merlot grapevine cultivated in high altitude region.}

The objective of this work is to compare the technological and phenolic maturation of the Merlot grapevine in a high altitude region of Santa Catarina, cultivated in soil covered by vegetation and an uncovered soil. The present work was carried out in the 2014/2015 harvest in a commercial vineyard located in São Joaquim city - Santa Catarina State. The experimental design was a randomized block design with four blocks and ten plants per block. The Merlot vineyard grafted on 'Paulsen 1103' was implanted in 2004. The treatments consisted in maintaining the crop line with natural vegetation cover 
and maintaining the crop line without vegetation cover. In order to maintain the crop line without vegetation cover, manual weeding was carried out from the phenological stage turned in color, this operation being repeated every 15 days until the moment of harvest. It was evaluated the technological maturation, phenolic berries, besides the mass of berries and bark/berry ratio. Effect of soil cover on the maturation of Merlot grapevine, on soil without vegetation cover in the growing line, increased ratio bark/berry, $\mathrm{pH}$ and anthocyanin. Therefore the soil without vegetation cover resulted in a better maturation of the Merlot grapevine cultivated in high altitude region.

Key words: Vitis vinifera L., Anthocyanins. Oenological quality. Wines of altitude.

\section{Introdução}

A região de elevada altitude de Santa Catarina (SC) apresenta uma a grande disponibilidade de radiação solar e baixas temperaturas noturnas, produzindo uvas com maior qualidade enológica (MALINOVSKI et al., 2016). Mesmo possuindo uma história recente (BRIGHENTI et al., 2013), a região já se destaca pela qualidade dos vinhos elaborados (FALCÃO et al. 2007; GRIS et al., 2010).

$\mathrm{Na}$ viticultura das regiões de elevadas altitudes de Santa Catarina, encontram-se condições edafoclimáticas, de elevada disponibilidade hídrica e solos com altos teores de matéria orgânica $(>3,5 \%)$, que promovem o excessivo crescimento vegetativo em detrimento do desempenho produtivo das videiras (MARCON FILHO et al., 2015). Além disso, verifica-se nessas regiões a utilização do porta-enxerto Paulsen 1103, com 72,1\% da área plantada até 2013 (VIANNA et al., 2016). O cultivo da videira enxertada com o porta-enxerto Paulsen 1103, em solos com alta disponibilidade de Nitrogênio, promove aumento no vigor dessa frutífera (MAFRA et al., 2011). Em condições de crescimento vegetativo excessivo a observa-se o sombreamento das folhas inferiores e cachos, o que pode interferir na composição das uvas (SPAYD et al., 2002). Podendo resultar em menor incidência de luz no interior do dossel, favorecendo o aparecimento de doenças fúngicas e afetar negativamente a composição da uva e de seu mosto (KELLER et al., 1998; DUCHÊNE; SCHNEIDER; GAUDILLÈRE, 2001; BRUNETTO et al., 2008).

A cobertura do solo pode promover competição, controlando a disponibilidade de nutrientes e água à videira (PÉREZ-BERMÚDEZ et al., 2016), atuando como alternativa para a redução do excesso de vigor vegetativo dessa frutífera, onde as condições edafoclimáticas são favoráveis à ocorrência desse problema (MONTEIRO; LOPES, 2007). De acordo com Rosseto et al. (2017), os sistemas de manejo de solo podem influenciar significativamente a temperatura do solo, pois alteram as condições da superfície do solo.

Deste modo, solos descobertos possuem a superfície escura e têm a capacidade de absorver maior energia e solos cobertos, de superfície clara, refletem maior quantidade de energia, o que ocorre com a palha que apresenta alta refletividade da radiação solar e condutividade térmica mínima (SALTON, 1991; CARNEIRO et al., 2013). Portanto a cobertura do solo pode influenciar a temperatura do solo, bem como as trocas de calor com o ambiente, podendo alterar a maturação da videira. Nesse contexto, o presente trabalho teve como objetivo comparar a maturação tecnológica e fenólica da videira Merlot em região de elevada altitude de Santa Catarina cultivada em com solo coberto por vegetação e um solo descoberto.

\section{Materiais e Métodos}

O experimento foi realizado na safra 2014/2015 em um vinhedo comercial (coordenadas $28^{\circ}$ $17^{\prime} 39^{\prime \prime} \mathrm{S}$ e $49^{\circ} 55^{\prime} 56^{\prime \prime} \mathrm{O}$, a $1.230 \mathrm{~m}$ de altitude), situado no munícipio de São Joaquim - Santa 
Catarina. O delineamento experimental utilizado foi o de blocos ao acaso, com quatro blocos e dez plantas por bloco.

O clima da região é classificado como 'Frio, Noites Frias e Úmido', Índice Heliotérmico de 1.714, precipitação pluvial média anual de $1.621 \mathrm{~mm}$ e a umidade relativa do ar média anual de $80 \%$ (TONIETTO; CARBONNAU, 2004). Os solos da região se enquadram nas classes Cambissolo Húmico, Neossolo Litólico e Nitossolo Háplico, desenvolvidos a partir de rocha riodacito e basalto (SOLOS, 2004).

Foi utilizado vinhedo da variedade Merlot enxertada sobre 'Paulsen 1103' implantado em 2004. O vinhedo se caracteriza por apresentar plantas espaçadas de $3,0 \times 1,5 \mathrm{~m}$, em filas dispostas no sentido $\mathrm{N}-\mathrm{S}$, conduzidas em espaldeira, em cordão esporonado duplo, a 1,2m de altura e cobertas com tela de proteção anti-granizo.

Os tratamentos consistiram em manter a linha de cultivo com cobertura vegetal natural e manter a linha de cultivo sem cobertura vegetal. Para manter a linha de cultivo sem cobertura vegetal foi realizado capina manual a partir do estágio fenológico virada de cor, sendo esta operação repetida a cada 15 dias até o momento da colheita, a fim de manter por todo o período, a linha de cultivo sem cobertura vegetal. Os tratos culturais (poda, desbrota, desfolha, desponte e tratamentos fitossanitários) foram realizados pela empresa de acordo com as recomendações dos responsáveis técnicos em todos os tratamentos.

No momento da colheita foram coletadas 150 bagas por parcela para análise da maturação tecnológica. As bagas foram esmagadas para separação do mosto e das cascas. A partir do mosto, foram determinados o teor de sólidos solúveis (SS), acidez titulável (AT) e pH, através de metodologias oficiais da Organização Internacional da Vinha e do Vinho (OIV, 2009). Para determinar o teor de sólidos solúveis (SS) utilizou-se um refratômetro digital para açúcar, modelo ITREFD-45, sendo os resultados expressos em ${ }^{\circ}$ Brix. A acidez total (AT) foi obtida através da titulação do mosto com solução alcalina padronizada de hidróxido de sódio $0,1 \mathrm{~N}$, utilizando como indicador o azul de bromotimol, sendo os resultados expressos em meq $\mathrm{L}^{-1}$. O potencial hidrogeniônico $(\mathrm{pH})$ foi registrado por meio de um potenciômetro marca Impac, após calibração em soluções tampões conhecidas de $\mathrm{pH} 4,0$ e 7,0. A massa da baga ( $\mathrm{g}$ ) foi calculada a partir do peso médio de 100 bagas. Também avaliou-se a relação entre a massa da casca e da baga, obtida pela equação: Relação casca/baga $(\%)=[\text { massa casca }(\mathrm{g}) / \text { massa baga }(\mathrm{g})]^{*} 100$.

Foram avaliadas a concentração de polifenóis totais e antocianinas. Para a obtenção das soluções-extrato foi utilizado a metodologia descrita por Marcon Filho et al. (2015). A concentração de polifenóis totais (PT) na casca foi determinada pelo método de espectrofotometria, descrito por Singleton e Rossi (1965), utilizando o reagente Folin-Ciocalteu e o ácido gálico como padrão. O teor de antocianinas na casca foi determinado pelo método de espectrofotometria, descrito por RibéreauGayon et al. (1998).

A cor foi determinada pelo método de espectrofotometria, descrito por Rizzon (2010). O extrato foi diluído na proporção 1:10 e analisado em espectrofotômetro nos comprimentos de onda de 420 $\mathrm{nm}, 520 \mathrm{~nm}$ e $620 \mathrm{~nm}$. A cor foi mensurada pelos parâmetros de intensidade e tonalidade de cor, obtida através das fórmulas: Intensidade $=420+520+620 \mathrm{~nm}$ e Tonalidade $=420 / 520 \mathrm{~nm}$.

Os dados foram submetidos à análise de variância (ANOVA) pelo 'Teste $F$ ' a $5 \%$ de probabilidade de erro. 


\section{Resultados e Discussão}

A massa de bagas, sólidos solúveis, acidez total e pH são apresentadas na Tabela 1. Para a variedade Merlot, verificou-se que não há influência da cobertura do solo na linha de cultivo para as variáveis massa de baga, sólidos solúveis e acidez total. No entanto, observou-se acréscimo do valor de $\mathrm{pH}$ nas bagas, quando as linhas de cultivos da videira Merlot estavam sem cobertura vegetal, apresentando 3,37, enquanto para o solo com cobertura vegetal observou-se valor de $\mathrm{pH}$ de 3,29 (Tabela 1). De acordo com Jackson (2014), para a elaboração de vinhos tintos de qualidade recomendam-se para o mosto, teores de sólidos solúveis acima de $20^{\circ}$ Brix, acidez total menor que 135 meq $\mathrm{L}^{-1}$ e $\mathrm{pH}$ menor que 3,5. Tais valores foram observados tanto para o solo com cobertura vegetal quanto sem para o solo sem cobertura vegetal.

Tabela 1 - Efeito da cobertura do solo na massa de bagas (gramas), sólidos solúveis ( ${ }^{\circ}$ Brix), acidez total (meq L ${ }^{-1}$ ) e pH durante a safra 2014/2015 em São Joaquim/Santa Catarina.

\begin{tabular}{ccccc}
\hline Tratamento & Massa baga & Sólidos Solúveis & Acidez total & pH \\
\hline Sem Cobertura & $g$ & ${ }^{\circ}$ Brix & $m e q L^{-1}$ & \\
Com Cobertura & $1,7 \mathrm{a}$ & $21,4 \mathrm{a}$ & $88,1 \mathrm{a}$ & $3,29 \mathrm{~b}$ \\
\hline CV (\%) & $1,7 \mathrm{a}$ & $21,9 \mathrm{a}$ & $87,6 \mathrm{a}$ & $3,37 \mathrm{a}$ \\
\hline
\end{tabular}

Observou-se efeito da cobertura do solo nas variáveis relação casca/baga (\%) e antocianinas $\left(\mathrm{mg} \mathrm{L}^{-1}\right)$. O solo descoberto resultou em acréscimo da relação casca/baga, indicando assim, maior volume de casca em relação a polpa. Em uvas destinadas à vinificação é desejada maior quantidade de casca, pois é nela que está a maior quantidade de compostos que incrementam a qualidade enológica do vinho, como antocianinas e taninos - em uvas para vinho tinto (OJEDA et al., 2004) - e precursores aromáticos, como terpenos - em uvas para vinhos brancos (KARAGIANNIS; ECONOMOU; LANARIDIS, 2000). Observou-se incremento no conteúdo de antocianinas, que está diretamente relacionada com aumento da relação casca/baga, pois na maioria das cultivares de uva, é restrita à película (KENNEDY et al., 2000). Enquanto o solo com cobertura vegetal apresentou $1354,1 \mathrm{mg} \mathrm{L}^{-1}$, as bagas provenientes do solo sem cobertura vegetal apresentou $1562,7 \mathrm{mg} \mathrm{L}^{-1} \mathrm{de}$ antocianinas (Tabela 2). A composição das antocianinas na uva depende primeiramente de fatores genéticos, porém a distribuição destes compostos durante a maturação da uva é ainda influenciada pelas condições climáticas e pelas características físico-químicas do solo (POMAR; NOVO; MASA, 2005).

Tabela 2 - Efeito da cobertura do solo na relação casca baga (\%), antocianinas (mg L ${ }^{-1}$ ), intensidade de cor $(420+520+620 \mathrm{~nm})$, tonalidade de cor (420/520) e polifenóis totais durante a safra 2014/2015 em São Joaquim/Santa Catarina.

\begin{tabular}{cccccc}
\hline Tratamento & $\begin{array}{c}\text { Relação } \\
\text { casca/baga }\end{array}$ & Antocianinas & $\begin{array}{c}\text { Intensidade de } \\
\text { cor }\end{array}$ & $\begin{array}{c}\text { Tonalidade } \\
\text { de cor }\end{array}$ & $\begin{array}{c}\text { Polifenóis } \\
\text { Totais }\end{array}$ \\
\hline $\begin{array}{c}\text { Sem } \\
\text { Cobertura }\end{array}$ & $19,1 \mathrm{~b}$ & $1354,1 \mathrm{~b}$ & $11,9 \mathrm{a}$ & $9,7 \mathrm{a}$ & $2099,5 \mathrm{a}$ \\
$\begin{array}{c}\text { Com } \\
\text { Cobertura }\end{array}$ & $26,2 \mathrm{a}$ & $1562,7 \mathrm{a}$ & $11,7 \mathrm{a}$ & $8,8 \mathrm{a}$ & $2283,2 \mathrm{a}$ \\
\hline $\mathbf{C V}(\%)$ & 8,99 & 5,11 & 13,11 & 6,03 & 6,17 \\
\hline
\end{tabular}

Apesar das antocianinas serem o componente fenólico responsável pela cor do vinho tinto (KENNEDY et al., 2000), não se observou efeito da cobertura do solo na intensidade de cor (420 + 
$520+620 \mathrm{~nm})$ e tonalidade de cor $(420 / 520 \mathrm{~nm})$ (Tabela 2). Tanto a intensidade de cor quanto a tonalidade são atributos importantes de um vinho, pois através de seu aspecto são obtidas informações sobre suas qualidades e possíveis defeitos (FREITAS, 2006).

Apesar do conteúdo de polifenóis totais das bagas provenientes do solo sem cobertura ter sido $8,04 \%$ maior que as bagas provenientes do solo com cobertura vegetal, não foram observadas diferenças estatisticamente significativas entre os tratamentos (Tabela 2). Os resultados observados com a variedade Merlot são superiores aos observados por Brighenti et al. (2017) estudando a variedade Merlot em região de elevada altitude.

\section{Conclusão}

A cobertura do solo influência a maturação tecnológica e fenólica da videira Merlot cultivada em região de elevada altitude.

A linha de cultivo da videira sem cobertura vegetal resulta em incremento do $\mathrm{pH}$, relação casca/baga e antocianinas totais, não influenciando a massa de baga, sólidos solúveis, acidez total, intensidade de cor, tonalidade de cor e polifenóis totais.

\section{Referências}

BRIGHENTI, A.F.; BRIGHENTI, E.; BONIN, V.; RUFATO, L. Caracterização fenológica e exigência térmica de diferentes variedades de uvas viníferas em São Joaquim, Santa Catarina - Brasil. Ciência Rural, Santa Maria, v.43, n.7, p.1162-1167, 2013.

BRIGHENTI, E.; CASAGRANDE, K.; CARDOSO, P.Z.; PASA, M.S.; CIOTTA, M.N.; BRIGHENTI, A.F. Total polyohenols contentes in diferente grapevine varieties in highlands of southern Brazil. Bio Web of Conferences, v.9, p. 1-5, 2017.

BRUNETTO, G.; BONGIORNO, C.L.; MATTIAS, J.L.; DEON, M.; MELO, G.W.B. de; KAMINSKI, J.; CERETTA, C.A. Produção, composição da uva e teores de nitrogênio na folha e no pecíolo em videiras submetidas à adubação nitrogenada. Ciência Rural, v.38, p.2622-2625, 2008.

CARNEIRO, R.G.; MOURA, M.A.L.; SILVA, V.P.R.; SILVA JÚNIOR, R.S.; ANDRADE, A.M.D.; SANTOS, A.B. Estudo da temperatura do solo em dois biomas florestais nos períodos, chuvoso e seco. Revista Brasileira de Geografia Física, v.6, p.1009-1022, 2013.

DUCHÊNE, E.; SCHNEIDER, C.; GAUDILLÈRE, J.P. Effects of nitrogen nutrition timing on fruit set of grapevine cv. Grenache. Vitis, v.40, p.45-46, 2001.

FALCÃO, L.D.; REVEL, G.; PERELLO, M.C.; MOUTSIOU, A.; ZANUS, M.C.; BORDIGNON-LUIZ, M.; A survey of seasonal temperatures and vineyard altitude influences on 2-methoxy-3-isobutylpyrazine, C13-norisprenoids, and the sensory profile of Brazilian Cabernet Sauvignon wines. Journal of Agricultural and Food Chemistry, v.55. p.3605-3612, 2007.

FREITAS, D.M. Variação dos compostos fenólicos e de cor dos vinhos de uvas (Vitis vinífera) tintas em diferentes ambientes. 2006. 56f. Tese (Doutorado em Agronomia) - Universidade Federal de Santa Maria, Santa Maria, 2006

GRIS, E.F.; BURIN, V.; BRIGHENTI, E.; VIEIRA, H.; BORDIGNON-LUIZ, M. Phenology and ripening of Vitis vinifera $L$. grape varieties in São Joaquim, southern Brazil: a new South American wine growing region. Ciencia e Investigación Agraria, v.37, p.61-75, 2010.

JACKSON, R. S. Wine Science: principles and applications. 751p. Elsevier, 4. Ed., 2014. 
KARAGIANNIS, S.; ECONOMOU, A.; LANARIDIS, P. Phenolic and volatile composition of wines made from Vitis vinifera cv. Muscat lefko grapes from the Island of Samos. Journal of Agricultural and Food Chemistry, v.48, p.5369-5375, 2000.

KELLER, M.; HRAZDINA, G. Interaction of nitrogen availability during bloom and light intensity during veraison. II. Effects on anthocyanin and phenolic development during grape ripening. American Journal of Enology and Viticulture, v.49, p.341-349, 1998.

KENNEDY, J.A.; TROUP, G.J.; PILBROW, J.R.; HUTTON, D.R.; HEWITT, D.; HUNTER, C.R.; RISTIC, R.; ILAND, P.G.; JONES, G.P. Development of seed polyphenols in berries from Vitis vinifera L. cv. Shiraz. Australian Journal of Grape and Wine Research, v.6, p.244-254, 2000.

MAFRA, S.H.; CASSOL, P.C.; MIQUELUTTI, D.J.; ERNANI, P.R.; GATIBONI, L.C.; FERREIRA, E.Z.; BARROS, M.; ZALAMENA, J.; GROHSKOPF, M.A. Atributos químicos do solo e estado nutricional de videira Cabernet Sauvignon (Vitis vinífera L.) na Serra Catarinense. Revista Ciências Agroveterinárias, v. 10, p. 44-53, 2011.

MALINOVSKI, L.I.; BRIGHENTI, A.F.; BORGHEZAN, M.; GUERRA, M.P.; SILVA, A.L.; PORRO, D.; STEFANINI, M.; VIEIRA, H.J. Viticultural performance of Italian grapevines in high altitude regions of Santa Catarina State, Brazil. Acta Horticulturae, v.1115, p.203-210, 2016.

MARCON FILHO, J. L.; HIPÓLITO, J.S.; MACEDO, T.A.; KRETZSCHMAR, A.A.; RUFATO, L. Raleio de cachos sobre o potencial enológico da uva 'Cabernet Franc' em duas safras. Ciência Rural, v.45, p.2150-2156, 2015.

MONTEIRO, A.; LOPES, C.M. Influence of cover crop on water use and performance of vineyard in Mediterranean Portugal. Agriculture, Ecosystems and Environment, v.12, p.336-342, 2007.

OJEDA, H.; DELOIRE, A.; WANG, Z.; CARBONNEAU, A. Determinación y control del estado hídrico de la vid: efectos morfológicos y fi siológicos de la restricción hídrica en vides. Viticultura/Enologia Professional, v.90, p.27-43, 2004.

OIV. Office International de la Vigne et du Vin. Recueil des Méthodes Internationales d'Analyse des Vins et des Moûts. Paris: Office International de la Vigne et du Vin, 2009.

PÉREZ-BERMÚDEZ, P.O.; OLMO, M.; GIL, J.; GARCÍA-FERRÍZ, L.; OLMO, C.; BOLUDA, R.; GAVIDIA, I. Cover crops and pruning in Bobal and Tempranillo vineyards have little influence on grapevine nutrition. Scientia Agricola, v.73, p.260-265, 2016.

POMAR, F.; NOVO, M.; MASA, A. Varietal differences among the anthocyanin profile of 50 red table grape cultivars studied by hight performance liquid chromatography. Journal Chromatograohy, v. 1094, p. 34-41, 2005.

RIBÉREAU-GAYON, P.; DONĖCHE, B.; DUBORDIEU, A.; LONVAUD, A. Traide d'enologie: microbiologie du vin: vinifications. Paris: Editorial Dunod, 1998. 185p..

RIZZON, L. A. (ed.) Metodologia para análise de vinho. Brasília: Embrapa Informação Tecnológica, , 2010. $120 \mathrm{p}$.

ROSSETO, R.E.; SANTOS, R.F.; SECCO, D.; CHANG, P. Temperatura do Solo e desenvolvimento de cultivos agrícolas. Journal of Agronomic Sciences, v.6, n. especial, p.95-103, 2017.

SALTON, J.C. Relações entre sistemas de preparo, temperatura e umidade do solo. 1991. 92p. Dissertação (Mestrado em Ciência do Solo) - Universidade Federal do Rio Grande do Sul, Porto Alegre, 1991

SINGLETON, V.L.; ROSSI JUNIOR, J.A. Colorimetry of total phenolics with phosphomolybdicphosphotungstic acid reagents. American Journal of Enology and Viticulture, v.16, p.144-158, 1965. 
SOLOS do Estado de Santa Catarina. Rio de Janeiro: Embrapa Solos, 2004. 726p. (Embrapa Solos. Boletim de pesquisa e desenvolvimento, 46 ).

SPAYD, S.E.; TARARA, J.M.; MEE, D.L.; FERGUSON, J.C. Separation of sunlight and temperature effects on the composition of Vitis vinifera cv. 'Merlot' berries. American Journal of Enology and Viticulture, v.53, p.171-182, 2002.

TONIETTO, J.; CARBONNEAU, A. A multicriteria climatic classification system for grape-growing regions worlwide. Agricultural and Forest Meteorology, v. 124, p. 81-97, 2004.

VIANNA, L.F.; MASSIGNAN, A.M.; PANDOLFO, C.; DORTZBACH, V.F.V. Caracterização agronômica e edafoclimáticas dos vinhedos de elevada altitude. Revista de Ciências Agroveterinárias, Lages, v.15, n.3, p.215-226, 2016. 